\title{
Safety of Anlotinib Capsules Combined with PD-1 Inhibitor Camrelizumab in the Third-Line Treatment of Advanced Non-Small-Cell Lung Cancer and Their Effect on Serum Tumor Markers
}

\author{
Yinhua Wang, ${ }^{1}$ Xiuhua Shi, ${ }^{1}$ Qinghua Qi, ${ }^{1}$ Bin Ye, ${ }^{1}$ and Zhaoling Zou $\mathbb{D}^{2}$ \\ ${ }^{1}$ Department of Radiotherapy, The Second People's Hospital of Wuhu City, Wuhu, 241000, Anhui Province, China \\ ${ }^{2}$ Department of Hematology, The Second People's Hospital of Wuhu City, Wuhu, 241000, Anhui Province, China \\ Correspondence should be addressed to Zhaoling Zou; zouzhaoling@whsph.org.cn
}

Received 4 November 2021; Revised 23 November 2021; Accepted 29 November 2021; Published 15 December 2021

Academic Editor: Kalidoss Rajakani

Copyright (c) 2021 Yinhua Wang et al. This is an open access article distributed under the Creative Commons Attribution License, which permits unrestricted use, distribution, and reproduction in any medium, provided the original work is properly cited.

\begin{abstract}
Objective. To explore the safety of anlotinib capsules combined with the PD-1 inhibitor (camrelizumab) in the third-line treatment of advanced non-small-cell lung cancer (NSCLC) and their effect on serum tumor markers. Methods. 88 patients with advanced NSCLC treated in the Oncology Department of our hospital from December 2018 to December 2019 were selected as research subjects and randomly and equally split into the single treatment group (STG) and combined treatment group (CTG). The levels of serum tumor markers after treatment were detected in both groups, and the incidence of adverse reactions during treatment was recorded. Results. Compared with the STG, CTG achieved obviously higher total effective rate $(P<0.05)$, lower total incidence of adverse reactions $(P<0.05)$, lower levels of serum tumor markers and average CFS score $(P<0.001)$, and higher average KPS score $(P<0.001)$. Conclusion. Application of anlotinib capsules combined with the PD-1 inhibitor (camrelizumab) in the thirdline treatment of advanced NSCLC can effectively reduce the levels of serum tumor markers and cancer fatigue degree of patients, with a better effect than that of simple anlotinib treatment. In addition, further research of the combined treatment is helpful to establish a better therapeutic regimen for patients with advanced NSCLC.
\end{abstract}

\section{Introduction}

With the changes in people's lifestyle and environment, the incidence of lung cancer in China has increased [1]. Radical resection of lung cancer is the best treatment for this disease. However, due to the occult onset of the cancer, most patients have entered the advanced stages when diagnosed, missing the best surgery time. Therefore, they turn to systemic chemotherapy, immunotherapy, or molecular targeted drug therapy [2-4]. Non-small-cell lung cancer (NSCLC) has a high incidence, accounting for about $65-80 \%$ of lung cancer, and the chemotherapy drugs are mostly cytotoxic drugs, which can fight against cancer and reduce tumors by damaging the DNA structure and affecting nucleic acid synthesis, but can trigger adverse reactions such as gastrointestinal reactions and myelosuppression because they do not have specificity for tumor cells and have a lethal effect on normal cells [5-7]. Third-line treatment refers to the treatment adopted after the failure of the second-line treatment. Generally, there are lesser drugs and effective treatment regimen for the third-line treatment, so the firstline treatment is the most crucial, which directly determines the prognosis of patient survival. Once the tumor resists to the first-line treatment, the subsequent treatment will obtain poorer effect, and therefore, it is necessary to actively explore highly efficient drugs used in the third-line treatment for prolonging the survival of NSCLC patients. In recent years, molecular targeted therapy has become a hot topic in treating NSCLC, which acts on a certain link of development and progression of tumor, such as inhibiting tumor angiogenesis and leading to tumor apoptosis. The advantage of molecular targeted therapy over conventional 
chemotherapeutic agents is that it does not cause toxic side effects such as myelosuppression and hair loss, so it is becoming the most acceptable treatment for medical workers and patients [8].

Anlotinib, a novel small-molecule and multitarget tyrosine kinase inhibitor independently developed in China, inhibits type III tyrosine kinase and platelet-derived growth factor receptor (PDGFR) to suppress tumor angiogenesis and growth, with the efficacy that has been proven in treating brain glioblastoma and metastatic renal cell carcinoma $[9,10]$. Camrelizumab, a humanized monoclonal antibody extracted from the hamster ovary cell line by recombinant technique, can bind to programmed death receptor-1 (PD-1), block the binding of PD-1 and programmed death receptor ligand 1 (PD-L1), reactivate $\mathrm{T}$ cells, produce sustained antitumor effect, and inhibit tumor growth, which has been confirmed in primary liver cancer with lung metastasis [11]. At present, no report has been found on the application of anlotinib capsules combined with the PD-1 inhibitor (camrelizumab) in the third-line treatment of patients with advanced NSCLC. This study adopted the combined treatment to explore its application value by observing the clinical manifestations and clinical indexes of patients.

\section{Materials and Methods}

2.1. General Information. Eighty-eight patients with advanced NSCLC treated in the Oncology Department of our hospital from December 2018 to December 2019 were selected as research subjects and randomly and equally split into the single treatment group (STG) and combined treatment group (CTG). Patients or legal guardians signed informed consent and volunteered to participate in the study.

\subsection{Inclusion and Exclusion Criteria}

2.2.1. Inclusion Criteria. The inclusion criteria were as follows: (1) all the enrolled patients met the diagnostic criteria of this disease in the Diagnostic and Therapeutic Norms for Primary Lung Cancer (2018 edition) [11], were confirmed by tissue biopsy and MRI with the clinical manifestations such as expectoration, dyspnea, and hemoptysis, and conformed to the stage IV staging criteria in International Standards for Lung Cancer Staging (8th edition) [12]; (2) the patients were no less than 18 years old, with the estimated survival period no less than 3 months; (3) the patients did not have contraindications in the drugs used in the study; and (4) the patients' physical condition allowed them to receive further treatment.

2.2.2. Exclusion Criteria. The exclusion criteria were as follows: (1) patients with abnormal coagulation and a risk of major bleeding; (2) patients complicated with failure of organs such as the liver, kidney, and heart, requiring supportive treatment or rescue; (3) patients with uncontrolled hypertension; (4) patients with a history of other malignancies.
2.3. Methods. All patients were treated with anlotinib hydrochloride (manufacturer: Chia Tai Tianqing Pharmaceutical Group Co., Ltd.; NMPA approval no. H20180003; specification: $12 \mathrm{mg} * 7$ capsules), with $12 \mathrm{mg} /$ time and 1 time/d for 2 consecutive weeks, followed by 1 week of rest. A treatment cycle included 3 weeks. Patients were discontinued until the occurrence of disease progression, intolerant adverse events, death, or refusal of treatment [13]. The dosage was reduced to $10 \mathrm{mg} / \mathrm{d}$ if grade 3 and above of anlotinib-related adverse events occurred during treatment (including (1) systolic blood pressure $\geq 180 \mathrm{mmHg}$; (2) severe skin changes such as bleeding, edema, blister, ulcer, peeling, hyperkeratosis, obvious pain, and limited self-care ability; and (3) $24 \mathrm{~h}$ urine protein excretion $\geq 3.5 \mathrm{~g}$ ) and to $8 \mathrm{mg} / \mathrm{d}$ if the patients remained intolerant. If still intolerant, the drug was discontinued.

CTG received anlotinib hydrochloride combined with camrelizumab. $200 \mathrm{mg}$ of camrelizumab (manufacturer: Suzhou Sheng Diya Biomedical Co., Ltd.; NMPA approval no. S20190027; specification: $200 \mathrm{mg} / \mathrm{vial}$ ) was injected by an intravenous drip, $200 \mathrm{mg} /$ time, 1 time/3 weeks. A chemotherapy cycle included 3 weeks. Drug administration was discontinued until the progression of the disease or the patients were intolerant.

2.4. Observation Indexes. The objective efficacy evaluation of tumors was as follows. The lesion size of the patients was recorded according to the results of CT, MRI, and other imaging examinations, and the therapeutic effect was evaluated using Response Evaluation Criteria in Solid Tumors (RECIST) [14]. The efficacy was complete response (CR, disappearance of all target lesions completely after treatment), partial response ( $\mathrm{PR}$, reduction of lesions $>50 \%$ and no new lesions), no change (NC, no significant improvement in clinical symptoms with tumor reduction between $25 \%$ and $50 \%$ ), and progression of the disease (PD, tumor increase $>25 \%$ or appearance of new lesions). Total effective rate $=(\mathrm{CR}+\mathrm{PR}) /$ total number of case$\mathrm{s} \times 100 \% ; 5 \mathrm{ml}$ of fasting elbow venous blood was collected after treatment and centrifuged at $3500 \mathrm{r} / \mathrm{min}$ for $10 \mathrm{~min}$. The levels of serum carcinoembryonic antigen (CEA), neuron-specific enolase (NSE), and cytokeratin-19 fragment (CYFRA 21-1) were detected by electrochemiluminescence. All the detection instruments were Roche cobas e 411 automatic electrochemiluminescence immunoassay analyzer (manufacturer: Shanghai Mojin Medical Device Co., Ltd.) and matching reagents (manufacturer: Shanghai Univ Biotechnology Co., Ltd.).

The German version of the Cancer Fatigue Scale (CFS) [15] was for evaluating the fatigue degree after treatment, including 15 items such as body, activity, attention, memory, and emotion, with each item scoring 5 points, a total score of 75 points. Higher scores represented more severe fatigue.

Karnofsky Performance Status (KPS) [16] was used to evaluate the physical condition of both groups before and after treatment, with the specific scoring criteria in Table 1.

The incidence of adverse reactions in both groups during treatment was analyzed, including hypertension, hand and foot syndrome, anemia, reactive cutaneous capillary hyperplasia, chest pain, and hemoptysis. 
TABLE 1: Karnofsky behavior scoring criteria.

\begin{tabular}{lc}
\hline Physical condition & Points \\
\hline Normal condition, no symptoms or signs & 100 \\
The patients could carry out normal activities, with mild symptoms and signs & 90 \\
The patients could carry out normal activities reluctantly, with some symptoms and signs & 80 \\
The patients could take care of themselves in daily life, but could not maintain normal life or activities & 70 \\
Sometimes, the patients needed help, but could generally take care of themselves & 60 \\
The patients needed others to look after them & 50 \\
The patients could not take care of themselves and needed special care & 40 \\
The patients could not take care of themselves at all & 30 \\
The patients had aggravated condition and required inpatient treatment & 20 \\
The patients were critically ill and close to death & 0 \\
Death &
\end{tabular}

2.5. Statistical Methods. The data were processed by SPSS 23.0 and graphed by GraphPad Prism 7 (GraphPad Software, San Diego, USA). Enumeration data were tested by $X^{2}$ and expressed as $n$ (\%), while measurement data were tested by $t$-test and expressed as mean $\pm \mathrm{SD}$. When $P<0.05$, the differences were statistically significant.

\section{Results}

3.1. Comparison of Baseline Data. No significant differences in baseline data such as sex ratio, average age, pathological types, and metastatic sites were observed between the two groups $(P>0.05$; Table 2$)$.

3.2. Comparison of Short-Term Efficacy. The total effective rate was remarkably higher in the CTG than in the STG $(P<0.05$; Table 3$)$.

3.3. Comparison of Serum Tumor Marker Levels. After treatment, the levels of various serum tumor markers in the CTG were obviously lower compared with the STG $(P<0.001$; Figure 1).

3.4. Comparison of CFS Scores after Treatment. The average CFS score after treatment in the CTG was obviously lower compared with the STG $(P<0.001$; Figure 2$)$.

3.5. Comparison of KPS Scores after Treatment. The average KPS score after treatment was markedly higher in the CTG than in the STG $(P<0.001$; Figure 3$)$.

3.6. Safety Comparison. The total incidence of adverse reactions in the CTG was lower compared with the STG $(P<0.05$; Table 4$)$.

\section{Discussion}

The incidence of lung cancer is increasing in China, accounting for the first and second place in male and female tumors, making it one of the malignant tumors with the highest mortality. In the early stage, the tumor does not involve the trachea, pleura, and bronchial mucosa and surrounding blood vessels, so there will be no symptoms of cough, chest pain, and hemoptysis, and therefore, the diagnosis of lung cancer is delayed to a great extent. A survey [17] has revealed that $75 \%$ of NSCLC patients are in advanced stages at diagnosis and miss the best surgery time. Although the existing chemotherapy regimens can play a certain therapeutic effect, most patients cannot tolerate the serious adverse reactions, and advanced NSCLC patients with multiline chemotherapy failure are difficult to benefit from chemotherapy again. As a novel micromolecule multitarget TKI (tyrosine kinase inhibitor) independently developed in China, anlotinib capsules are convenient for oral administration, with little adverse reactions and good patient tolerance in clinical trials, which are expected to become the typical drug in the third-line treatment of NSCLC in China [18]. In a clinical study [19], 166 patients with advanced soft tissue sarcoma were treated with anlotinib, and the results showed that the 12 -week disease progression-free rate was $68 \%$, progression-free survival time was 5.6 months, and the overall median survival time was 12 months. Camrelizumab is a humanized monoclonal antibody obtained by the recombinant technique. Since this drug has shown good survival benefits in the single-arm phase II clinical trials of typical Hodgkin's lymphoma, it is used to treat recurrent or refractory classical Hodgkin's lymphoma after second-line systemic chemotherapy [20].

Among the treatment methods of various tumor diseases, the combined therapy has more advantages for patients. Lou et al. [21] pointed out in the study that the survival curves of patients treated with single drug and chemotherapy were crossed, suggesting that some patients cannot benefit from single-drug therapy. Visser et al. [22] found an obvious difference in the survival curves at the early stage between patients treated with combined therapy and those treated with monotherapy, demonstrating that the combined therapy is the better treatment pattern for cancer. In this paper, anlotinib capsules combined with the PD-1 inhibitor (camrelizumab) were adopted to treat advanced NSCLC, and the results demonstrated that the total clinical effective rate of the CTG was 93.18\% (41/44), which was higher than $75.00 \%$ (33/ 44) of the STG, revealing that the combined treatment could significantly improve the efficacy of patients with advanced 
TABle 2: Comparison of baseline data $(n=44)$.

\begin{tabular}{|c|c|c|c|c|}
\hline Items & STG & CTG & $X^{2} / t$ & $P$ \\
\hline Gender & & & 0.188 & 0.665 \\
\hline Male & $27(61.36 \%)$ & $25(56.82 \%)$ & & \\
\hline Female & $17(38.64 \%)$ & $19(43.18 \%)$ & & \\
\hline Average age (mean $\pm S D$, years old) & $64.38 \pm 7.28$ & $64.56 \pm 7.24$ & 0.116 & 0.908 \\
\hline $\mathrm{BMI}\left(\right.$ mean $\left.\pm \mathrm{SD}, \mathrm{kg} / \mathrm{m}^{2}\right)$ & $21.25 \pm 1.26$ & $21.32 \pm 1.23$ & 0.264 & 0.793 \\
\hline \multicolumn{5}{|l|}{ Pathological types } \\
\hline Adenocarcinoma & $24(54.55 \%)$ & $26(59.09 \%)$ & 0.185 & 0.667 \\
\hline Squamous cell carcinoma & $17(38.64 \%)$ & $14(31.82 \%)$ & 0.448 & 0.503 \\
\hline Others & $3(6.82 \%)$ & $4(9.09 \%)$ & 0.155 & 0.694 \\
\hline \multicolumn{5}{|l|}{ Metastatic sites } \\
\hline Liver & $12(27.27 \%)$ & $10(22.73 \%)$ & 0.246 & 0.620 \\
\hline Pleura & $8(18.18 \%)$ & $11(25.00 \%)$ & 0.612 & 0.434 \\
\hline Lymph nodes & $19(43.18 \%)$ & $16(36.36 \%)$ & 0.441 & 0.507 \\
\hline Bones & $5(11.36 \%)$ & $7(15.91 \%)$ & 0.389 & 0.533 \\
\hline Smoking history & & & 0.279 & 0.597 \\
\hline Yes & $34(77.27 \%)$ & $36(81.82 \%)$ & & \\
\hline No & $10(22.73 \%)$ & $8(18.18 \%)$ & & \\
\hline Drinking history & & & 0.196 & 0.658 \\
\hline Yes & $29(65.91 \%)$ & $27(61.36 \%)$ & & \\
\hline No & $15(34.09 \%)$ & $17(38.64 \%)$ & & \\
\hline \multicolumn{5}{|l|}{ Marital status } \\
\hline Married & $40(90.91 \%)$ & $39(88.64 \%)$ & 0.124 & 0.725 \\
\hline Unmarried & $1(2.27 \%)$ & $3(6.82 \%)$ & 1.048 & 0.306 \\
\hline Divorced & $3(6.82 \%)$ & $2(4.55 \%)$ & 0.212 & 0.645 \\
\hline \multicolumn{5}{|l|}{ Education level } \\
\hline University & $6(13.64 \%)$ & $4(9.09 \%)$ & 0.451 & 0.502 \\
\hline Middle school & $12(27.27 \%)$ & $16(36.36 \%)$ & 0.838 & 0.360 \\
\hline Primary school & $26(59.09 \%)$ & $24(54.55 \%)$ & 0.185 & 0.667 \\
\hline Residence & & & 0.049 & 0.826 \\
\hline Urban area & $17(38.64 \%)$ & $16(36.36 \%)$ & & \\
\hline Rural area & $27(61.36 \%)$ & $28(63.64 \%)$ & & \\
\hline
\end{tabular}

TABle 3: Comparison of short-term efficacy $(n(\%))$.

\begin{tabular}{lcccccc}
\hline Group & $n$ & CR & PR & NC & PD & Total effective rate \\
\hline CTG & 44 & $25(56.82 \%)$ & $16(36.36 \%)$ & $2(4.55 \%)$ & $1(2.27 \%)$ & $93.18 \%(41 / 44)$ \\
STG & 44 & $19(43.18 \%)$ & $14(31.82 \%)$ & $7(15.91 \%)$ & $4(9.09 \%)$ & $75.00 \%(33 / 44)$ \\
$X^{2}$ & & & & & 5.436 \\
$P$ & & & & & $<0.05$ \\
\hline
\end{tabular}

NSCLC compared with the simple treatment, with positive significance for prolonging the survival time and improving the prognosis. CYFRA $21-1$ is an epithelial cell characteristic marker, mainly distributed in unilayer epithelial cells and pseudostratified epithelial cells, so this serum tumor marker is a good indicator of tumors of epithelial origin. Comparison of serum tumor marker levels after treatment in this study showed that the levels were lower in the CTG than in the STG $(P<0.001)$, suggesting that the combined treatment was more conducive to the treatment of patients with tumors. The presence of cancer fatigue is related to the course of the disease and chemotherapy on the one hand and the experience of persistent subjective fatigue on the other hand, which can not only lead to a decrease in the quality of life of patients but also promote the progression of the condition, leading to discontinue treatment. The study results confirmed that the mean CFS score after treatment was significantly lower in the CTG than in the STG $(P<0.001)$, indicating that the combination modality could reduce their cancer fatigue and contribute to the improvement of quality of life. In terms of adverse reactions, the PD-1 inhibitor (camrelizumab) not only benefits the majority of cancer patients but also brings immune-related adverse reactions because it can involve multiple systems in the human body [23]. Among them, reactive cutaneous capillary hyperplasia is the most common adverse drug reaction of camrelizumab, with the clinicopathologic features of dermal capillary increase and proliferation of capillary endothelial cells. Its current pathogenesis remains unclear, which may be due to the imbalance between angiogenesis promoters and inhibitors [24]. The study showed that only 3 patients had this symptom during treatment in the CTG but did not endanger life, and the patients recovered spontaneously after a period of drug withdrawal. 


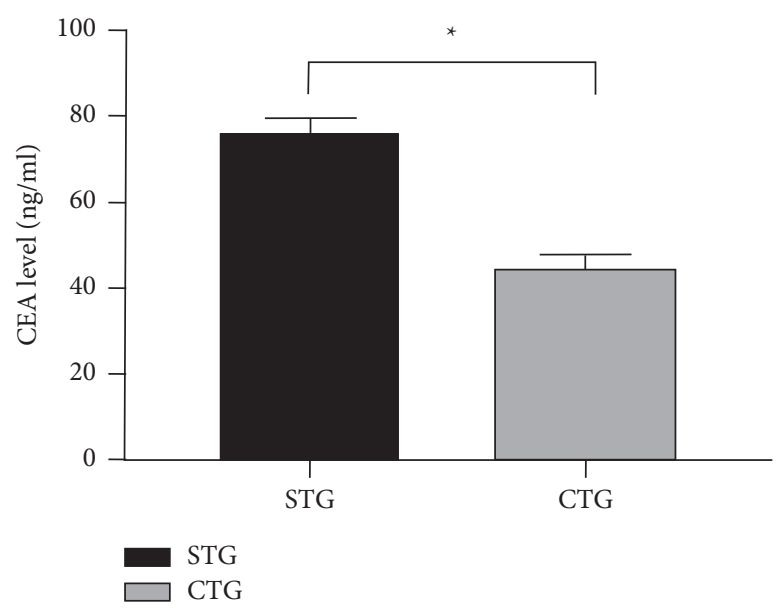

(a)

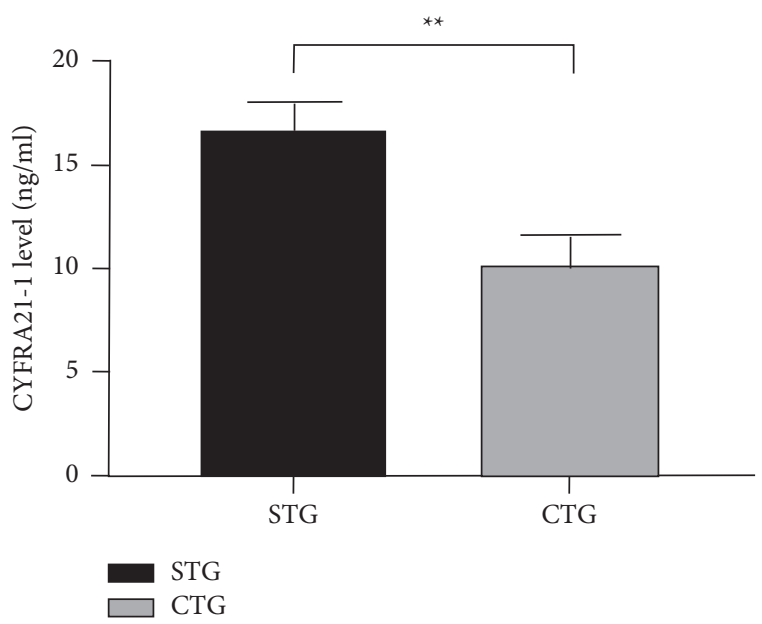

(b)

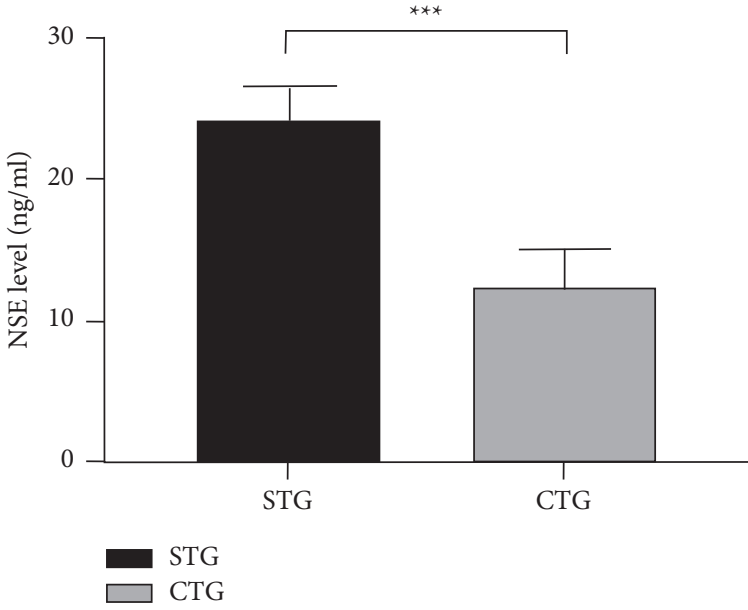

(c)

FIGURE 1: Comparison of serum tumor marker levels after treatment (mean \pm SD). (a) Comparison of CEA levels. The abscissa was STG and CTG, and the ordinate was the CEA level $(\mathrm{ng} / \mathrm{ml})$. The CEA levels in the STG and CTG were $76.26 \pm 3.64 \mathrm{ng} / \mathrm{ml} \mathrm{and} 44.35 \pm 3.48 \mathrm{ng} / \mathrm{ml}$. *indicated a remarkable difference in the CEA levels between the two groups after treatment $(t=42.032, P<0.001)$. (b) Comparison of CYFRA 21-1 levels. The abscissa was STG and CTG, and the ordinate was the CYFRA 21-1 level (ng/ml). The CYFRA 21-1 levels in the STG and CTG were $16.74 \pm 1.35 \mathrm{ng} / \mathrm{ml}$ and $10.32 \pm 1.28 \mathrm{ng} / \mathrm{ml} .{ }^{* *}$ indicated a remarkable difference in the CYFRA $21-1$ levels between the two groups after treatment $(t=22.891, P<0.001)$. (c) Comparison of NSE levels. The abscissa was STG and CTG, and the ordinate was the NSE level (ng/ml). The NSE levels in the STG and CTG were $24.16 \pm 2.43 \mathrm{ng} / \mathrm{ml}$ and $12.54 \pm 2.56 \mathrm{ng} / \mathrm{ml} .{ }^{* * *}$ indicated a remarkable difference in the NSE levels between the two groups after treatment $(t=21.837, P<0.001)$.

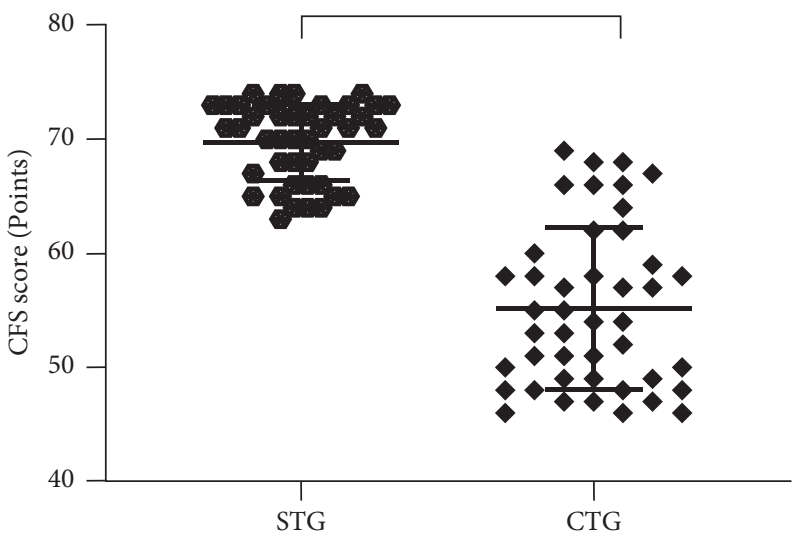

Figure 2: Comparison of CFS scores after treatment (mean \pm SD). Note: the abscissa was STG and CTG, and the ordinate was the CFS score (points). The average CFS scores after treatment in the STG and CTG were $69.75 \pm 3.38$ and $55.16 \pm 7.09 .{ }^{*}$ indicated an obvious difference in the average CFS scores after treatment between the two groups $(t=12.322, P<0.001)$. 


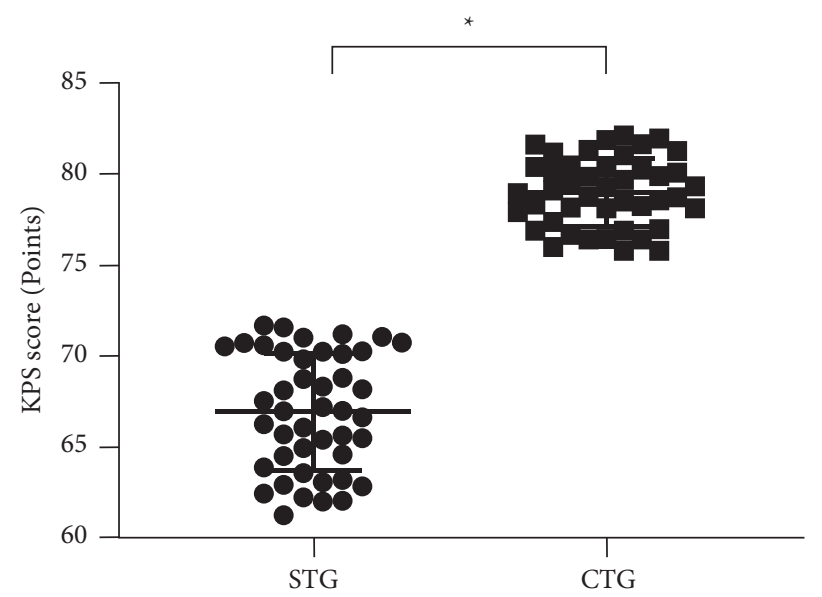

FIGURE 3: Comparison of KPS scores after treatment (mean \pm SD). Note: the abscissa was STG and CTG, and the ordinate was the KPS score (points). The average KPS scores in the STG and CTG were 66.96 \pm 3.21 and $79.02 \pm 1.86 .{ }^{*}$ indicated a remarkable difference in the average KPS scores between the two groups after treatment $(t=21.563, P<0.001)$.

TABLE 4: Comparison of the incidence of adverse reactions $(n(\%))$.

\begin{tabular}{lccccccc}
\hline Group & Hypertension & $\begin{array}{c}\text { Hand-foot } \\
\text { syndrome }\end{array}$ & Anemia & Reactive cutaneous capillary hyperplasia & Chest pain & Hemoptysis & $\begin{array}{c}\text { Total } \\
\text { incidence }\end{array}$ \\
\hline STG & $3(6.82)$ & $3(6.82)$ & $2(4.55)$ & $0(0.00)$ & $2(4.55)$ & $3(2.27)$ & $29.55 \%(13 / 44)$ \\
CTG & $1(2.27)$ & $0(0.00)$ & $1(2.27)$ & $3(6.82)$ & $0(0.00)$ & $0(0.00)$ & $11.36 \%(5 / 44)$ \\
$X^{2}$ & & & & & 4.470 \\
$P$ & & & & & $<0.05$ \\
\hline
\end{tabular}

The study also has some limitations. For example, the limited number of cases and the small sample size may lead to bias in the experimental results. In addition, it is impossible to analyze the median survival period of patients and long-term efficacy of the combined treatment. Therefore, multicenter studies with a larger sample size are needed in the future to further provide the best treatment regime for NSCLC patients.

In conclusion, the combination of anlotinib capsules and PD-1 inhibitor (camrelizumab) is a reliable scheme in the third-line treatment of advanced NSCLC, which greatly reduces the serum tumor markers of patients, with high safety and clinical application value.

\section{Data Availability}

The data used to support the findings of this study are available from the corresponding author upon reasonable request.

\section{Conflicts of Interest}

The authors declare no conflicts of interest.

\section{References}

[1] C. Aggarwal, R. B. Cohen, E. Yu et al., "Etirinotecan pegol (NKTR-102) in third-line treatment of patients with metastatic or recurrent non-small-cell lung cancer: results of a phase II study," Clinical Lung Cancer, vol. 19, no. 2, pp. 157-162, 2018.
[2] Y. Yang, K. Chen, Y. Zhou, Z. Hu, S. Chen, and Y. Huang, "Application of serum microRNA-9-5p, 21-5p, and 223-3p combined with tumor markers in the diagnosis of non-smallcell lung cancer in Yunnan in southwestern China," OncoTargets and Therapy, vol. 11, pp. 587-597, 2018.

[3] Z. Q. Chen, L. S. Huang, and B. Zhu, "Assessment of seven clinical tumor markers in diagnosis of non-small-cell lung cancer," Disease Markers, vol. 2018, Article ID 9845123, 7 pages, 2018.

[4] M. Zhuo, H. Chen, T. Zhang et al., "The potential predictive value of circulating immune cell ratio and tumor marker in atezolizumab treated advanced non-small cell lung cancer patients," Cancer Biomarkers: Section A of Disease Markers, vol. 22, pp. 467-476, 2018.

[5] Y. Zhang, B. Jia, J. Li, and X. Xu, "Anlotinib in treatment of an elderly patient with recurrent advanced SCLC," Tumori Journal, vol. 106, no. 6, pp. NP36-NP40, 2020.

[6] H. Deng, B. Li, L. Li et al., "Clinical observation of crizotinib in the treatment of ALK-positive advanced non-small cell lung cancer," Pathology, Research \& Practice, vol. 215, no. 12, Article ID 152695, 2019.

[7] H. Ito, Y. Matsuo, S. Ohtsu et al., "Impact of histology on patterns of failure and clinical outcomes in patients treated with definitive chemoradiotherapy for locally advanced nonsmall cell lung cancer," International Journal of Clinical Oncology, vol. 25, no. 2, pp. 274-281, 2020.

[8] H. Kawachi, M. Tamiya, S. Ishii et al., "Association between metastatic sites and first-line pembrolizumab treatment outcome for advanced non-small cell lung cancer with high PD-L1 expression: a retrospective multicenter cohort study," Investigational New Drugs, vol. 38, pp. 211-218, 2020. 
[9] A. Qin, R. Rengan, S. Lee et al., "A pilot study of atezolizumab plus hypofractionated image guided radiation therapy for the treatment of advanced non-small cell lung cancer," International Journal of Radiation Oncology, Biology, Physics, vol. 108, no. 1, pp. 170-177, 2020.

[10] Y. Ozaki, S. Muto, H. Takagi et al., "Tumor mutation burden and immunological, genomic, and clinicopathological factors as biomarkers for checkpoint inhibitor treatment of patients with non-small-cell lung cancer," Cancer Immunology, Immunotherapy, vol. 69, no. 1, pp. 127-134, 2020.

[11] V. A. Papadimitrakopoulou, J. Y. Han, M. J. Ahn et al., "Epidermal growth factor receptor mutation analysis in tissue and plasma from the AURA3 trial: osimertinib versus platinum-pemetrexed for T790M mutation-positive advanced non-small cell lung cancer," Cancer, vol. 126, no. 2, pp. 373-380, 2020.

[12] S. Khozin, R. A. Miksad, J. Adami et al., "Real-world progression, treatment, and survival outcomes during rapid adoption of immunotherapy for advanced non-small cell lung cancer," Cancer, vol. 125, pp. 4019-4032, 2019.

[13] S. Y. Zheng, H. J. Cui, H. Duan et al., "The efficacy and safety of immune checkpoint inhibitors in non-small cell lung cancer patients of different age groups: a meta-analysis," Clinical and Translational Oncology, vol. 22, no. 7, pp. 1146-1154, 2020.

[14] R. Sara Elena, P. Fabiana, B. Melissa, B Giacomo, M Michele, and B Sebastiano, "Prognostic and predictive molecular biomarkers in metastatic renal cell carcinoma patients treated with immune checkpoint inhibitors: a systematic review," Expert Rev Mol Diagn, vol. 20, pp. 169-185, 2020.

[15] B. P. Singh, J. L. Marshall, and A. R. He, "Workup and management of immune-mediated colitis in patients treated with immune checkpoint inhibitors," The Oncologist, vol. 25, no. 3, pp. 197-202, 2020.

[16] H. J. M. Smit, J. Aerts, M. van den Heuvel et al., "Effects of checkpoint inhibitors in advanced non-small cell lung cancer at population level from the National Immunotherapy Registry," Lung Cancer, vol. 140, pp. 107-112, 2020.

[17] Y. Sakata, K. Kawamura, K. Ichikado et al., "The association between tumor burden and severe immune-related adverse events in non-small cell lung cancer patients responding to immune-checkpoint inhibitor treatment," Lung Cancer, vol. 130, pp. 159-161, 2019.

[18] X. Li, X. Zhao, M Abbas et al., "Comparative effectiveness study of single high-dose cisplatin with fractionated doses cisplatin in first-line therapy for treatment-naive Chinese patients with advanced non-small-cell lung cancer," Current Problems in Cancer, vol. 43, no. 6, Article ID 100466, 2019.

[19] M. A. Gubens, L. V. Sequist, J. P. Stevenson et al., "Pembrolizumab in combination with ipilimumab as second-line or later therapy for advanced non-small-cell lung cancer: KEYNOTE-021 cohorts D and H," Lung Cancer, vol. 130, pp. 59-66, 2019.

[20] Z. Gao, Y. Shang, X. Wang et al., "Application of circulating tumor DNA for dynamic monitoring of advanced non-small cell lung cancer treatment response: an open-label, multicenter, prospective, observational study protocol," Thoracic Cancer, vol. 10, no. 5, pp. 1310-1315, 2019.

[21] Y. Lou, J. A. Marin-Acevedo, P. Vishnu et al., "Hypereosinophilia in a patient with metastatic non-small-cell lung cancer treated with antiprogrammed cell death 1 (anti-PD-1) therapy," Immunotherapy, vol. 11, no. 7, pp. 577-584, 2019.

[22] S. Visser, S. L. W. Koolen, P. de Bruijn et al., "Pemetrexed exposure predicts toxicity in advanced non-small-cell lung cancer: a prospective cohort study," European Journal of Cancer, vol. 121, pp. 64-73, 2019.

[23] Z. Wei, X. Yang, X. Ye et al., "Camrelizumab combined with microwave ablation improves the objective response rate in advanced non-small cell lung cancer," Journal of Cancer Research and Therapeutics, vol. 15, pp. 1629-1634, 2019.

[24] M. Gusella, F. Pasini, D. Caruso et al., "Clinical outcomes of oral metronomic vinorelbine in advanced non-small cell lung cancer: correlations with pharmacokinetics and MDR1 polymorphisms," Cancer Chemotherapy and Pharmacology, vol. 83, pp. 493-500, 2019. 